\title{
Strain Variation Among Candida Species: Application of Various Typing Methods to Study the Epidemiology and Pathogenesis of Candidiasis in Hospitalized Patients
}

Candida spp, particularly $C$ albicans and $C$ tropicalis, are pervasive pathogens capable of causing acute and recurrent local disease in otherwise healthy individuals ${ }^{1-7}$ and life-threatening systemic infection in immunocompromised patients. ${ }^{1-3,8-11}$ Despite the recognition of the clinical importance of these organisms, there clearly are deficiencies in our ability to prevent, diagnose, and treat these infections, particularly in immunocompromised patients. $1-3,9,10,12$ In large part that may be due to our lack of knowledge concerning the epidemiology and pathogenicity of Candida spp. The application of several newer methods for subspeciating isolates of $C$ albicans and other Candida spp may improve our understanding of the epidemiology and pathogenesis of candidal infections. The remainder of this editorial will review the clinical significance of candidal infection in immunocompromised patients and the importance of strain differentiation and phenotypic variation in our understanding of the epidemiology and pathogenesis of candidal infections.

Infection continues to be a major cause of morbidity and mortality following bone marrow transplantation $9,10,13$ and therapy of patients with hematologic malignancies. ${ }^{1,8,10}$ Although bacterial infections are clearly important, opportunistic infections due to Candida spp are of particular concern and appear to have increased markedly over the past two to three decades. ${ }^{1-3,9}$ Systemic candidiasis is reported to occur in $20 \%$

From the Veterans Administration Medical Center and the Department of Pathology, Universily of Iowa College of Medicine, Iowa City, Iowa.

Address reprint requests to Michael A. P faller, MD, Department of Pathology, 273 MRC, University of Iowa College of Medicine, Iowa City, IA 52242. to $40 \%$ of patients with hematologic malignancies $1-3,10$ and in approximately $28 \%$ of patients who receive bone marrow transplants. ${ }^{1-3}$ Improvements in life support capabilities and advances in cytotoxic, immunosuppressive, and antibacterial therapies have all combined to prolong the survival of these patients, with a resulting substantial increase in the number at risk for developing severe local and systemic candidal infections.

Systemic candidal infection may involve many diverse anatomical sites including lung, liver, spleen, kidney, and cardiovascular and central nervous systems. ${ }^{1-3}$ Currently, it is thought that the first step in the development of systemic candidiasis is colonization of the gastrointestinal tract or oropharynx by Candida spp. Candidal infection may then disseminate by one of three major routes: 1) direct vascular invasion via an intravascular catheter ${ }^{1-3,9,14}$; 2) vascular invasion following disruption of the mucosal surface of the colonized oropharyngeal or gastrointestinal tract ${ }^{2,14-18}$; or 3) persorption of organisms through the intact gastrointestinal mucosa and into the bloodstream due to sheer numbers of colonizing organisms. ${ }^{15,16,18-20}$

Of primary importance in the establishment of systemic candidal infection is the immunologic status of the host. Virtually all patients with systemic candidiasis have severe defects in their host defense system and most commonly are neutropenic or manifest impaired neutrophil function. $1-3,9$

Despite the recognition of Candida as an important pathogen in the severely immunocompromised patient, the results of prevention and therapy of systemic candidiasis have been dismal. The reported mortality of systemic candidiasis in patients with hematologic malignancies or bone marrow transplantation ranges from $60 \%$ to $90 \%$, despite treatment with amphotericin $B^{1-3,8-13}$ 
Attempts at prophylaxis with oral or systemic administration of antifungal agents have been unsuccessful in preventing invasive disease, despite a reduction in the extent of local colonization. ${ }^{1-3,8-13,17}$ These results suggest that extensive local colonization and superinfection by Candida spp may be an overly simplistic view of the pathogenesis of systemic candidiasis.

One of the major problems in the prevention and therapy of candidiasis in immunocompromised patients is the difficulty in diagnosing infection versus colonization in these extremely complicated patients. ${ }^{10,21,22}$ The clinical signs and symptoms associated with both local and systemic candidiasis are well known but are nonspecific and generally not helpful in distinguishing bacterial infection from candidal infection. ${ }^{1-3}$ The laboratory diagnosis is also both insensitive and nonspecific. $1-3,9,10$ Although histologic examination of tissue obtained by biopsy remains extremely useful in establishing the diagnosis of invasive disease, it is frequently not practical in patients at high risk for candidal infection, due to severe thrombocytopenia or impaired coagulation in these patients. ${ }^{1-3}$

In addition to the diagnostic problems noted above, problems exist in interpreting cultures obtained from a variety of other body sites due to the propensity for $\mathrm{Can}$ dida spp, particularly $C$ albicans, to colonize these sites without causing infection. ${ }^{2,14,21,22}$ Studies by Sandford et $\mathrm{al}^{22}$ document the fact that despite colonization by $C$ albicans in the respiratory, genitourinary, and gastrointestinal tract of the majority of patients with hematologic malignancies or bone marrow transplantation, only a small percentage ever develop invasive disease due to this organism. In contrast, $58 \%$ to $100 \%$ of patients colonized by $C$ tropicalis develop systemic infection and thus are at significantly greater risk of developing a systemic infection due to $C$ iropicalis. Although these data suggest that $C$ tropicalis may be more invasive than $C$ albicans, particularly following cytotoxic chemotherapy, the reasons for this increased virulence are unclear. ${ }^{11,18}$

Additional investigation into the epidemiology and pathogenesis of candidal infection in the immunocompromised patient seems warranted in order to provide information that may be exploited in our efforts to control these pervasive pathogens. Unfortunately, the prevailing theme in many of the previous studies dealing with the epidemiology and pathogenicity of Candida spp has been to treat each species as a single organism with a single set of phenotypic traits. As a result we know very little about the degree of strain variation within species and its relation to the ability of an organism to colonize or invade a specific body site in a given patient. Differences among strains within species have been recognized and include the ability to adhere to various cellular and plastic surfaces, ${ }^{14-16,23,24}$ ability to respond to various pathophysiologic changes in the host with increased growth or mycelial formation, ${ }^{14,18,19,25,26}$ development of resistance to antifungal agents, $9,11,12,14,27$ and in the case of $C$ albicans, differences in serotype ${ }^{6,14,28-31}$ and the ability to produce proteases ${ }^{29,32,33}$ and phospholipase. $23,29,34$ However, these differences have not been fully exploited to differentiate strains for epidemiologic purposes and have not been incorporated into a unified explanation of the pathogenicity of the organisms. The delineation of specific phenotypic (or genotypic) profiles of Candida spp would provide the basis for studies designed to answer important epidemiologic and pathogenetic questions in immunocompromised patients: 1) the role of strain variation in chronic colonization and recurrent infection; 2) the site of origin of organisms causing invasive disease; and 3) the frequency and mechanism of patient-topatient or hospital staff-to-patient spread of Candida isolates involved in nosocomial infections. In addition, such studies could serve to identify potential virulence markers in clinical isolates that could be further characterized using molecular, immunologic, and genetic techniques, and could be tested in an animal model of invasive disease as well. Finally, strain differentiation would be potentially useful in interpreting culture results and establishing rational prophylactic and chemotherapeutic protocols, particularly if one or more phenotypes were found more predictive of invasive disease than others. Based on such data, the need for isolation precautions in certain patients could be addressed and their efficacy subsequently tested.

Although they have not yet been applied to the investigation of candidiasis in the immunocompromised patient, several different typing systems have recently been developed for the purpose of subspeciating isolates of $C$ albicans and other species of Candida. ${ }^{6,29,31}$ These typing methods include the serological method of Hasenclever and Mitchell, ${ }^{28}$ the biotyping system of Odds and Abbott, ${ }^{35,36}$ the resistogram system of McCreight and Warnock, ${ }^{37}$ the killer system of Polonelli et al, ${ }^{38}$ and DNA restriction fragment analysis. ${ }^{39}$ Of these typing systems, three have been applied extensively in investigation of the epidemiology of oral and vaginal candidiasis. The serologic method, which divides $C$ albicans into two serogroups, group A and group B, is extremely limited in its ability to delineate strains of $C$ albicans infection. ${ }^{29,31}$ The biotyping system of Odds and Abbott ${ }^{35}$ utilizes a set of nine tests that together permit 512 potential biotypes of $C$ albicans to be distinguished. These tests include acid and salt tolerance tests, proteinase production, two resistance tests, and four assimilation tests. Recently this system was expanded to include a total of 14 tests that allowed further delineation of $C$ albicans strains and, in addition, subspeciation of $C$ tropicalis and other species of Candida as well. ${ }^{36}$ Of the 512 potential biotypes that can be distinguished with the nine-test battery, about 160 have so far been encountered among patients with candidiasis. ${ }^{6.29,31,36}$ The application of this typing system to the study of vaginal candidiasis has resulted in the following conclusions $5.6,29$ :

1) In general, a given patient carries the same biotype of $C$ albicans at different sites (oral, rectal, vaginal) and at different times, regardless of symptomatology.

2) Patients with recurrent disease are infected with the same biotype of $C$ albicans at each recurrence.

3) Most vaginal infections are endogenous.

4) Venereal transmission of $C$ albicans can occur.

Warnock and co-workers $7,31,37$ used a resistogram method for strain differentiation and obtained results similar to those of Odds. The resistogram method is based on differences in resistance to five selected chemi- 
cals incorporated into agar media. The chemicals included in the most recent modification are sodium selenite, boric acid, cetrimide, sodium periodate, and silver nitrate. The method permits the discrimination of 32 potential resistotypes of $C$ albicans, each of which differs from the other 31 resistotypes in its reaction to at least one of the five chemicals used. The method has been found to be reproducible to within one test difference. ${ }^{31,37}$

Polonelli and co-workers ${ }^{38}$ utilized killer factors for $C$ albicans typing. Killer factors are toxins produced by certain yeasts that kill other yeasts within the same genus or within other genera. The killer characteristic is determined by a double stranded extranuclear RNA. Polonelli et $\mathrm{al}^{38}$ have selected eight Hansenula strains and one Pichia species for use in this system that theoretically permits 512 different strains of $C$ albicans to be distinguished. The method has been extended to differentiate strains of other species of Candida including $C$ tropicalis, $C$ parapsilosis, and $C$ pseudotropicalis. ${ }^{40}$ Although promising, this method has not been widely applied in clinical or epidemiologic studies of Candida spp. ${ }^{6,29,31}$

The application of DNA-typing methods to study strain variation and epidemiology of Candida spp is very promising. This method has recently been described by Scherer and Stevens ${ }^{39}$ and relies on DNA extraction, digestion of the genomic DNA with restriction endonuclease, and electrophoresis, resulting in DNA of various restriction lengths producing, in a gel, unique patterns that are analogous to "fingerprints." Similar approaches have been successful in using plasmid, viral, or bacterial DNA patterns as epidemiologic tools. Scherer and Stevens ${ }^{39}$ have described their initial results with this method and found that several Candida species could be separated into mutually exclusive groups and subgroups. The method was reproducible and relatively easy to perform. Many polymorphisms were noted.

The findings described above provide a base for further epidemiologic studies using the various phenotypic and DNA-typing methods. Taken together, these findings suggest that 1) usable typing systems do exist that can reliably delineate strains of clinical $C$ albicans isolates; 2) considerable diversity exists among $C$ albicans, thereby making epidemiologic studies feasible; 3) $C$ albicans can be passed from person to person; and 4) infection due to $C$ albicans is usually of endogenous origin with the same strain colonizing several anatomic sites. Thus, it appears that the typing systems described above will be useful tools in studying the epidemiology of $C$ albicans, and possibly other species of Candida, in immunocompromised patients, as well as those with vaginal candidiasis.

The limitation of the studies cited above is that, for the most part, the study populations were limited to outpatients with oral or vaginal candidiasis. Studies of severely immunocompromised, hospitalized patients are clearly indicated and may provide additional insights into the epidemiology and pathogenesis of candidal infections. In a more recent study, Burnie, Odds and co-workers ${ }^{4}$ used biotyping, colony morphology, and serotyping to investigate an outbreak of systemic candidiasis in an intensive care unit. Using this approach, they found that one particular strain of $C$ albicans was capable of causing systemic candidiasis as a result of cross-infection between patients and staff. Control of the outbreak was dependent on identifying the causative strain and prevention of crossinfection. They were able to demonstrate 1 ) an association between a specific biotype and virulence; 2) the usefulness of strain differentiation in the study of Candida colonization and infection in hospitalized patients; and 3) an exogenous source for candidal infection in severely ill patients. These data illustrate the practical utility of strain differentiation in nosocomial infection control efforts and, furthermore, suggest that differences in virulence may exist among clinical isolates of $C$ albicans.

Although the above studies provide some evidence for the diversity of Candida species as pathogens, the general concept still prevails of these organisms as extremely low virulence pathogens dependent entirely on the status of the host for their disease-causing potential. ${ }^{14,22,29}$ This concept of pathogenicity does not fully explain the ability of Candida spp to resist antifungal therapy, $9,11,12,14,27$ evade the immune response, ${ }^{30}$ and invade such a diverse spectrum of anatomic sites. ${ }^{1-3}$ Likewise, it does not explain why some immunosuppressed patients develop invasive infection while others who are equally immunosuppressed and colonized with $C$ albicans, do not become infected. ${ }^{22}$ Additional, carefully designed prospective studies of hospitalized patients are essential to provide new insight into the basis for such diverse biologic and pathologic behavior in $C$ albicans and other species of Candida. The information gained from studies employing useful typing methods will result in improved methods of prevention, diagnosis, and therapy, as well as increased understanding of the pathogenesis of candidal infections in immunosuppressed patientş.

\section{REFERENCES}

1. Bodey GP: Candidiasis in cancer patients. $A m$ J Med 1984; 77:13-19

2. Bodey GP, Fainstein V: Systemic candidiasis, in Bodey GP, Fainstein V (eds): Candidiasis. New York, Raven Press, 1985, pp 135-168.

3. Bodey GP: Fungal infection and fever of unknown origin in neutropenic patients. Am / Med 1986; 80(suppl 5C):112-119.

4. Burnie JP, Odds F, Lee W, et al: Outbreak of systemic Candida albicans on an intensive care unit caused by cross-infection. BrMed J 1985; 290:746-748.

5. Odds FC: Genital candidosis. Clin Exp Dermatol 1982; 7:345-354.

6. Stenderup A: Ecology of yeast and epidemiology of yeast infections. Acta Derm Venereol 1986; 121:27-37.

7. Warnock DW, Speller DCE. Milne JD. et al: Epidemiological investigation of patients with vulvovaginal candidosis. Br J Vener Dis 1979; 55:357-361.

8. Gold JWM: Opportunistic fungal infections in patients with neoplastic disease. Am J Med 1984; 76:458-463.

9. Horn R, Wong B, Kiehn TE, et al: Fungemia in a cancer hospital: Changing frequency, earlier onset, and results of therapy. Rev Infect Dis 1985; 7:646-655.

10. Maksymiuk AW, Thongprosert S, Hopfer R, et al: Systemic candidiasis in cancer patients. Am J Med 1984; 77:20-27.

11. Wingard JR. Merz WG, Saral R: Candida tropicalis: A major pathogen in immunocompromised patients. Ann Intern Med 1979; 91:539-543.

12. Medoff G, Kobayashi GS: Strategies in the treatment of systemic fungal infections. N Engl J Med 1980; 302:145-155.

13. Winston D], Gale RP, Meyer DV, et al: Infectious complications of human bone marrow transplantation. Medicine 1979; 58(1):31.

14. Shepherd MG, Poulter RTM, Sullivan PA: Candida albicans: Biology, genetics, and pathogenicity. Anmu Rev Microbiol 1985; 39:579-614.

15. Kennedy MJ, Volz PA: Ecology of Candida albicans gut colonization: Inhibition of Candida adhesion. colonization and dissemination from the gastrointestinal tract by bacterial antagonism. Infect Immun 1985; 49:654-663.

16. Klotz SA, Drutz 1)J. Harrison JL, et al: Adherence and penetration of vascular endothelium by Candida yeasts. Infect Immun I983; 42:374-384

17. Schimpff $\mathrm{SC}$, Young $V$, Greene $\mathrm{WH}$, et al: Origin of infection in acute nonlymphocytic leukemia: Significance of hospital acquisition of potential pathogens. Ann Intern Med 1972; 77:707-714.

18. Wingard JR. Dick JD. Merz WG, et al: Pathogenicity of Candida tropicalis and Candida albicans after gastrointestinal inoculation in mice. Infect Immun 1980; 29:808-813. 
19. Kennedy MJ, Volz PA: Dissemination of yeasts after gastrointestinal inoculation in antibiotic-treated mice. Sabouraudia 1983; 21:27-33.

20. Kennedy M, Volz PA: Effect of various antibiotics on gastrointestinal colonization and dissemination by Candida albicans. Sabouraudia 1985; 23:265-273.

21. Kiehn TE, Edwards FF, Armstrong D: The prevalence of yeasts in clinical specimens from cancer patients. Am / Clin Pathol 1980; 73:518-521.

22. Sandford GR, Merz WG, Wingard JR, et al: The value of surveillance cultures as predictors of systemic fungal infections. J Infect Dis 1980; 142:503-509.

23. Barrett-Bee $\mathrm{K}$, Hayes $\mathrm{Y}$, Wilson RG, et al: A comparison of phospholipase activity. cellular adherence and pathogenicity of yeasts. $J$ Gen Microbiol 1985; 131:1217-1221

24. Wingard JR, Dick JD, Merz WG, et al: Differences in virulence of clinical isolates of Candida tropicalis and Candida albicans in mice. Infect Immun 1982; 37:833-836.

25. Hubbard MJ, Markie I), Poulter RrM: Isolation and morphological characterization of a mycelial mutant of Candida albicans. J Bacteriol 1986; 165:61-65.

26. Odds FC: Morphogenesis in Candida albicans. CRC: Crit Rev Microbiol 1985; 12:45-93.

27. Defever KS, Whelan WL., Rogers AL, et al: Resistance to 5-fluorocytosine in Candida albicuns: The frequency of partially resistant strains among clinical isolates. Antimicrob Agents Chemother 1982; 22:810-815.

28. Hasenclever HR, Michell WO: Antigenic studies of Candida I: Observation of two antigenic groups in Candida albicans. J Bacteriol 1961; 82:570-573.

29. Odds FC: Biotyping of medically important fungi, in McGinnis MR (ed): Current Topics in Medical Mycology, Vol 1. New York, Springer-Verlag, 1985, pp $15.5-17 !$.
30. Poulain D, Hopewood V, Vernes A: Antigenic variability of Candida albicans. CRC Crit Rev Microbiol 1985; 12:223-270.

31. Warnock DW: Typing of Candida albicans. I Hosp Infect 1984; 5:244-252.

32. MacDonald F, Odds FC: Inducible proteinase of Candida albicans in diagnostic serology and in the pathogenesis of systemic candidosis. J Med Microbiol 1980; $13: 423-435$

33. MacDonald F, Odds FC: Virulence for mice of a proteinase-secreting strain of Candida albicans and a proteinase-deficient mutant. I Gen Microbiol 1983; 129:431-438

34. Price MF, Wilkinson ID. Gentry LO: Plate method for detection of phospholipase activity in Candida albicans. Sabouraudia 1982; 20:7-14.

35. Odds FC, Abbott AB: A simple system for the presumptive identification of Candida albicans and differentiation of strains within the species. Sabouraudia $1980 ; 18: 301-317$.

36. Odds FC, Abbott AB: Modification and extension of tests for differentiation of Candida species and strains. Sabouraudia 1983; $21: 79-81$.

37. McCreight MC, Warnock DW: Enhanced differentiation of isolates of Candida albicans using a modified resistogram method. Mykosen 1982; 25:589-598.

38. Polonelli L, Archibusacci C. Sestito M. et al: Killer system: A simple method for differentiating strains of Candida albicans. J Clin Microbiol 1983; 17:774-780.

39. Scherer S, Stevens DA: Application of DNA typing methods to epidemiology and taxonomy of Candida species. J Clin Microbiol 1987; 25:675-679.

40. Morace G, Archibusacci C, Sestito M. et al: Strain differentiation of pathogenic yeasts by the killer system. Mycopathologia 1984; 84:81-85. 